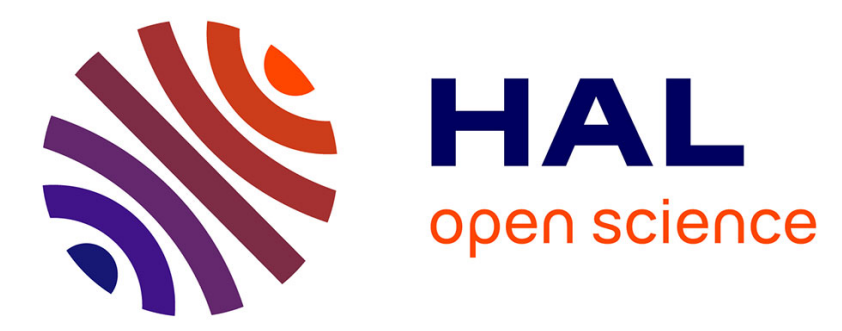

\title{
Heuristic models for diffraction by some simple micro-objects
}

Gaid Moulin, François Goudail, Pierre Chavel, Dengfeng Kuang

\section{To cite this version:}

Gaid Moulin, François Goudail, Pierre Chavel, Dengfeng Kuang. Heuristic models for diffraction by some simple micro-objects. Journal of the Optical Society of America. A Optics, Image Science, and Vision, 2009, 26 (4), pp.767. 10.1364/JOSAA.26.000767 . hal-00555910

\section{HAL Id: hal-00555910 \\ https://hal-iogs.archives-ouvertes.fr/hal-00555910}

Submitted on 6 Apr 2012

HAL is a multi-disciplinary open access archive for the deposit and dissemination of scientific research documents, whether they are published or not. The documents may come from teaching and research institutions in France or abroad, or from public or private research centers.
L'archive ouverte pluridisciplinaire HAL, est destinée au dépôt et à la diffusion de documents scientifiques de niveau recherche, publiés ou non, émanant des établissements d'enseignement et de recherche français ou étrangers, des laboratoires publics ou privés. 


\title{
Heuristic models for diffraction by some simple micro-objects
}

\author{
Gaid Moulin, ${ }^{1}$ François Goudail, ${ }^{1, *}$ Pierre Chavel, ${ }^{1}$ and Dengfeng Kuang ${ }^{2}$ \\ ${ }^{1}$ Laboratoire Charles Fabry de l'Institut d'Optique, CNRS, Univercité Paris-Sud, \\ Campus Polytechnique, RD 128, 91127 Palaiseau, France \\ ${ }^{2}$ Key Laboratory of Opto-Electronic Information Science and Technology \\ (Ministry of Education), Institute of Modern Optics, Nankai University, Tianjin 300071, China \\ *Corresponding author: francois.goudail@institutoptique.fr
}

Received October 22, 2008; accepted December 28, 2008;

posted January 28, 2009 (Doc. ID 103146); published March 12, 2009

\begin{abstract}
Electromagnetic optics is normally expected to be the only appropriate approach to describe structures with features of just a few wavelengths. But in some cases, these structure can be well described by simple heuristic arguments relying on geometrical optics and on diffraction by known elementary primitives. Such an approach allows a better understanding of the involved physical phenomena and reduces the computation time. We investigate the case of a microcomponent with a triangular section by using two approximate models with increasing complexity and explore their limits as the size of the structure decreases. Results are compared with a rigorous electromagnetic approach and discussed on the basis of near-field and far-field diffraction patterns. (C) 2009 Optical Society of America

OCIS codes: $260.1960,260.211,050.1970$.
\end{abstract}

\section{INTRODUCTION}

Elementary physics courses teach that diffraction can be approached, to a good first approximation, by fairly elementary concepts and techniques. In that context, the wave equation is satisfied in free-space propagation, but at some points in space Maxwell's equations are not rigorously satisfied. Indeed, the accuracy of such elementary methods is known to be limited when the size of the structure is of the same order of the wavelength. It is well known that Maxwell's equations are, in the framework of classical macroscopic physics, the only complete description of light propagation in any given geometrical configuration. The whole field of electromagnetic optics is based on solving Maxwell's equations through appropriate algorithmic methods. It has been developed to solve grating problems involving fine structures as well as micro-optics and nano-optics questions such as photonic bandgap materials, plasmonics, or metamaterials [1]. In fact, the number of situations of practical interest to the community where the numerical methods of electromagnetic optics are required is steadily increasing. Nevertheless, it is always useful to the physicist when numerical solutions can be supplemented with a heuristic description of what is happening in the structure. Simple arguments that retain the essence of physical phenomena and provide an intuitive understanding of their behavior are essential to explore them further and to exploit them in applications. They can also alleviate the numerical modeling burden.

In this work we wish to contribute exploring, on one specific case of interest, how far an elementary approach can be pushed in the modeling of diffraction by fine structures and still give results very close to the electromagnetic solution. Previous contributions in the same direction include [2-6]. Performing a similar comparison on blazed gratings, Swanson [2] has undertaken to "fill the void between scalar and electromagnetic theories." $\mathrm{He}$ thus showed that the standard "scalar theory" can be extended by ray tracing through the finite thickness of the grating profile and by taking into account, for example, the shadowing effects. The results of the scalar theory are significantly improved by that extension. Other studies of the shadowing effect on blazed grating include $[7,8]$. Based on the "rigorous coupled wave technique," Pommet et al. [3] have determined regions of validity of scalar diffraction theory in the case of binary gratings, both singlelevel and multilevel. In [4], Bendickson et al. rigorously express diffraction by specific cylindrical objects using a Green formulation and systematically relate the most common approximate methods to the former. They also express how those approximate methods depart from an accurate prediction of field distribution at the focal line of a cylindrical lens. Testorf [5] and Singer et al. [6] analyzed the departure between rigorous diffraction and the thin element approximation common in Fourier optics as a perturbative problem. The first perturbative term, identified to the first Born approximation, provided insight into specific grating problems. A Green function formulation of the perturbation approach can be found in [9]. It has also been shown by several authors $[10,11]$ that semirigorous models combining rigorous and approximate scalar calculations can give results very close to the fully rigorous method with a reduction of computation time in the case of binary gratings. Ufimtsev [12] has developed a physical theory of diffraction that combines ray optics and diffraction effects at discontinuities of the surface of scattering objects. It has been applied mainly in acoustics and electromagnetics for predicting the backscattering of impenetrable objects. 
We will study in this paper a weakly diffracting triangular structure with feature size close to the wavelength. We shall consider two levels of improvement of the thin element approximation, and we base our discussion on comparisons of far-field diffraction patterns, and also on the near-field structure, which gives a physical insight in the phenomena involved. In Section 2, we start from the elementary "scalar" approximation in the form known as the "thin element approximation" and then gradually introduce more elaborate models that increase the degree of precision, comparing their results with those of the "rigorous coupled-wave analysis" method (RCWA) [13,14], which in this case can be considered as a reference from electromagnetic optics, as good convergence is always achieved. Specifically, improving the "thin element approximation," we first investigate a more refined, still purely geometric approach. Then we empirically introduce the effects of diffraction at discontinuities of the structure. In Section 3, we discuss the limits of our model, and we offer some concluding comments in Section 4.

\section{COMPARISON OF MODELS}

In this work we are focusing on one-dimensional triangular structures surrounded by air. They are thus characterized by their height $h$, the length of their base $L$, and their index $n$. We assume that they are illuminated by a plane wave of wavelength $\lambda=0.5 \mu \mathrm{m}$ with a TE polarization at normal incidence (Fig. 1). The structure of interest is isolated in free space. However, to use a RCWA grating solver as our electromagnetic method of reference, we will consider that the structure is periodically repeated. In all cases, the introduced periodicity $\Lambda$ has been selected to be quite large so that no interaction occurs between two neighboring triangles. Typically, $\Lambda=8 L$ proved sufficient. That procedure has two practical effects: it introduces diffraction orders, and when computing the far-field diffraction we shall exhibit only the diffraction efficiencies of the various orders. Also, it provides an expedient way to normalize the diffraction pattern with respect to the incident plane wave.

Our purpose is to determine both the near-field map and the far-field diffraction pattern of this structure. Provided that no other sources exist apart from the incident wave on the input plane of the structure (Fig. 1), the wave equation applied on the components of the field (the TE component in the case of this article) insures that the solution in the output bottom half space is simply given by

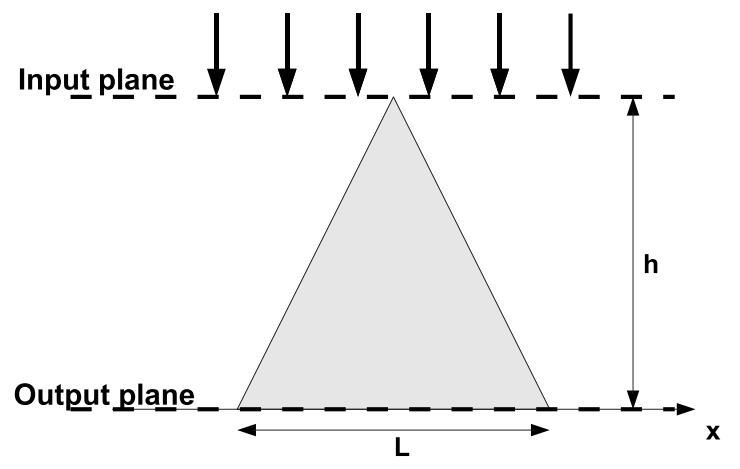

Fig. 1. Triangular structure under study. the plane-wave decomposition of the field in the output plane. Obviously, the evanescent waves remain confined close to this output plane. Therefore, we shall compute the diffraction efficiencies. The efficiency corresponding to the $m^{\text {th }}$ order is given by

$$
\eta_{m}=\frac{\left|a_{m}\right|^{2}}{\left|E_{\text {inc }}\right|^{2}},
$$

with $a_{m}$ the $m^{\text {th }}$ coefficient of the Fourier transform of the electric field at the output of the structure and $E_{\text {inc }}$ the incident field amplitude. Each order is diffracted under a diffraction angle given by the grating equation, which can be expressed in our case as

$$
\sin \left(\theta_{m}\right)=\frac{m \lambda}{\Lambda}
$$

We shall represent the far-field diffraction pattern by plotting the efficiencies as a function of the diffraction angles. It can be noticed that the scale of the efficiency values does not have any direct meaning. Indeed, this value is linked to the periodicity $\Lambda$, which is only a computation parameter without any physical interest in the problem considered in this article.

The main issue will be to compute this field with approximate scalar methods with increasing complexity. RCWA computations are carried out using Reticolo software for grating analysis [developed by J. P. Hugonin and P. Lalanne at Institut d'Optique, Orsay, France (2005)].

\section{A. Thin Element Approximation (TEA)}

A scalar model frequently used for modeling phase components is the thin element approximation (TEA) $[3,15,16]$. As is clearly explained, for example, in [15] Section 5.1, it consists of computing the amplitude and the phase of the field at the output of the structure as if light passed straight through it (Fig. 2). The field in the output plane can thus be expressed as

$$
E(x)=\exp \left(\frac{2 i \pi}{\lambda}\left[n_{\text {air }} l_{1}(x)+n l_{2}(x)\right]\right),
$$

where $l_{1}(x)$ and $l_{2}(x)$ correspond respectively to the distances traveled by light in the air and in the medium of index $n$.

The amplitude and the phase of the output field given by the TEA model are plotted Figs. 3(a) and 4(a), respectively, together with those given by the RCWA method. In the TEA model, as we do not consider any partial reflec-

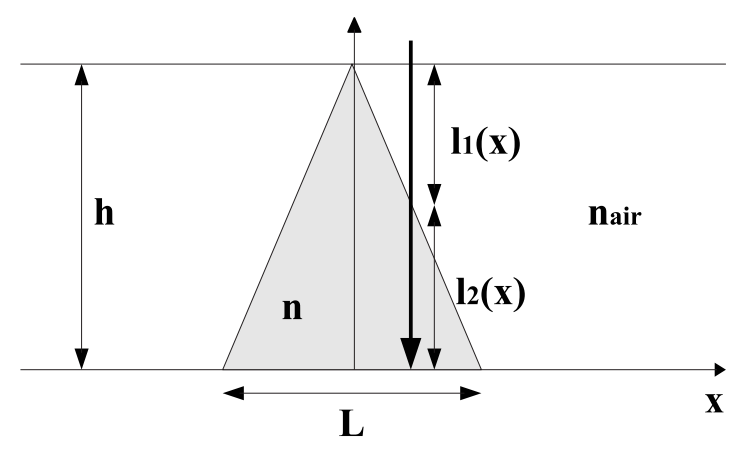

Fig. 2. TEA model. 


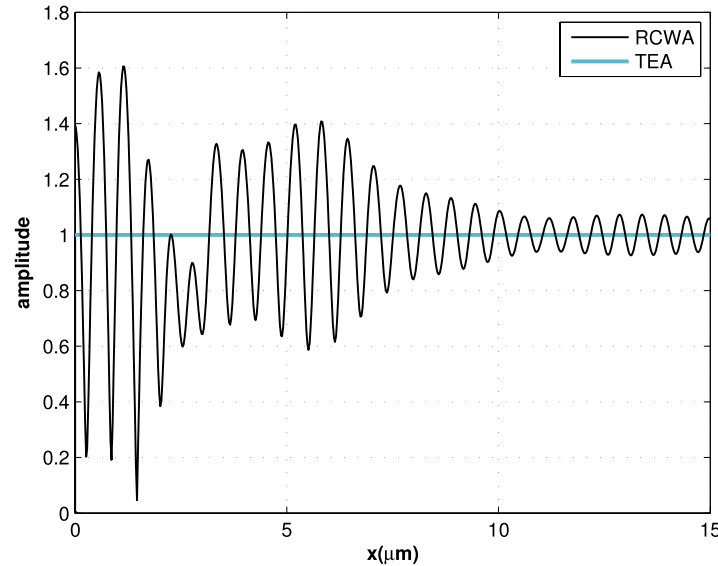

(a)

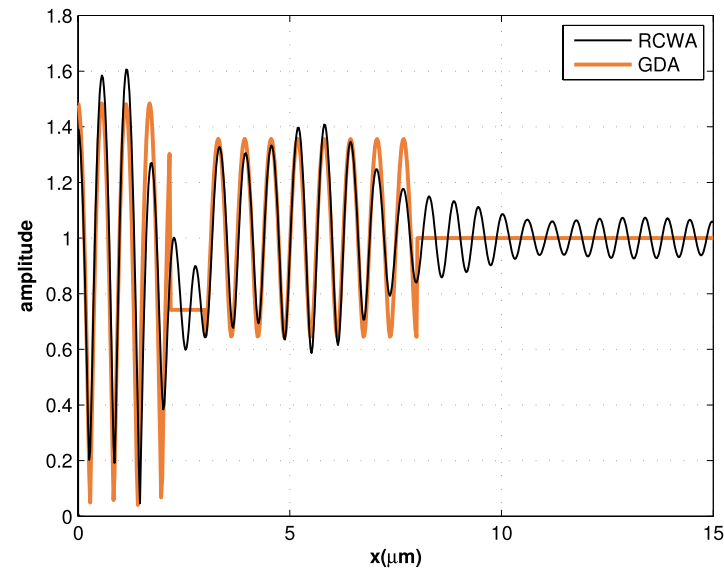

(b)

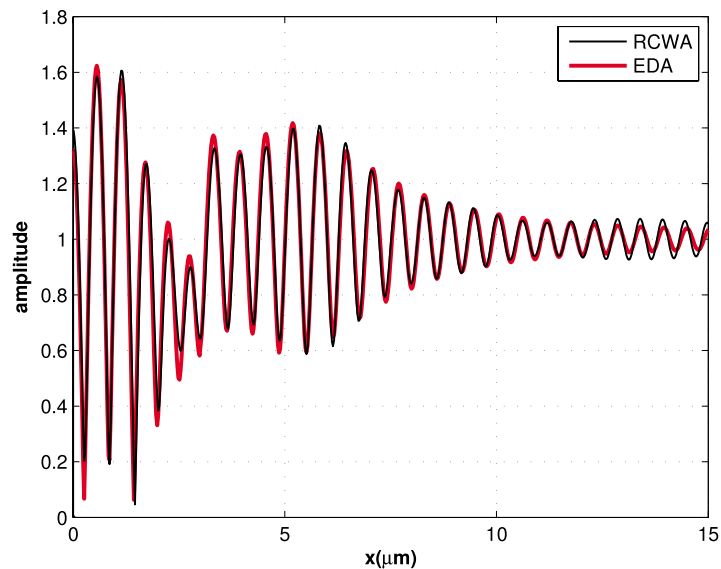

(c)

Fig. 3. (Color online) Amplitude of the field for a structure with $h=6 \mu \mathrm{m}, L=6 \mu \mathrm{m}, n=1.3$, and $\Lambda=50 \mu \mathrm{m}$ computed with (a) TEA, (b) GDA, and (c) EDA models and compared with the RCWA model.

tion of light on the sides of the triangle, the amplitude of the field is equal to unity everywhere, and the variation of the phase delay though the triangle is linear as a function of $x$. With these values of the parameters, many details of the real field are not grasped with this model. Obviously,

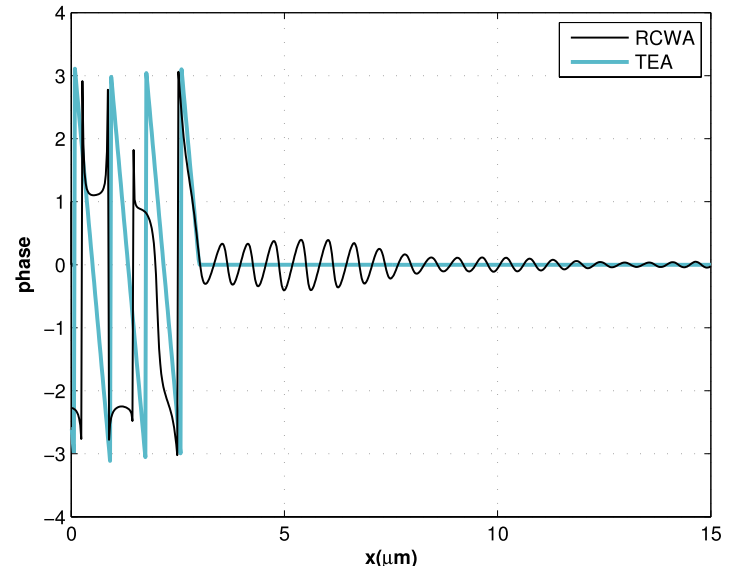

(a)

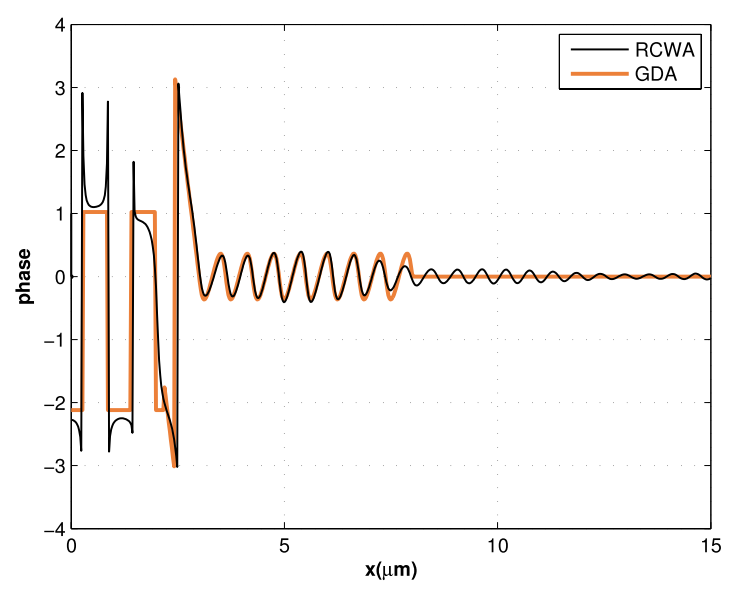

(b)

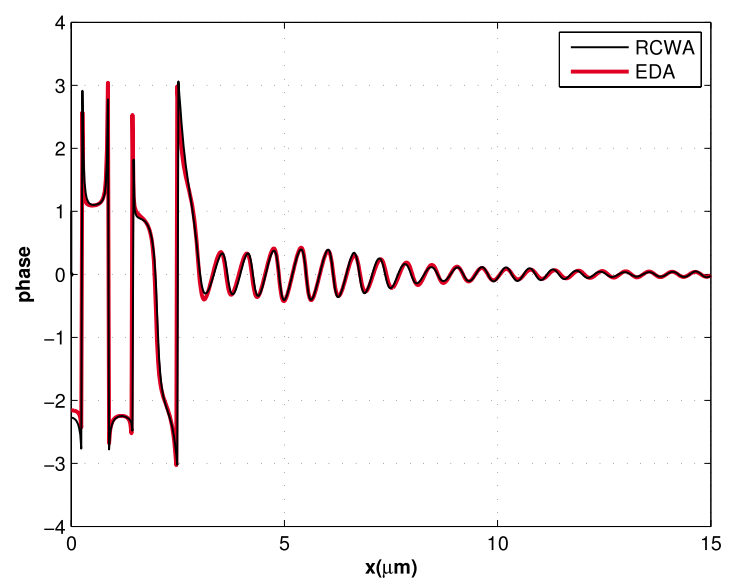

(c)

Fig. 4. (Color online) Phase of the field for a structure with $h$ $=6 \mu \mathrm{m}, L=6 \mu \mathrm{m}, n=1.3$, and $\Lambda=50 \mu \mathrm{m}$ computed with (a) TEA, (b) GDA, and (c) EDA models and compared with the RCWA model.

a better agreement would be found by leaving the height $h$ constant and increasing the base length $L$.

The far-field diffraction pattern computed with the TEA and RCWA models are represented in Fig. 5(a). This figure shows that the results of the TEA model are quite 


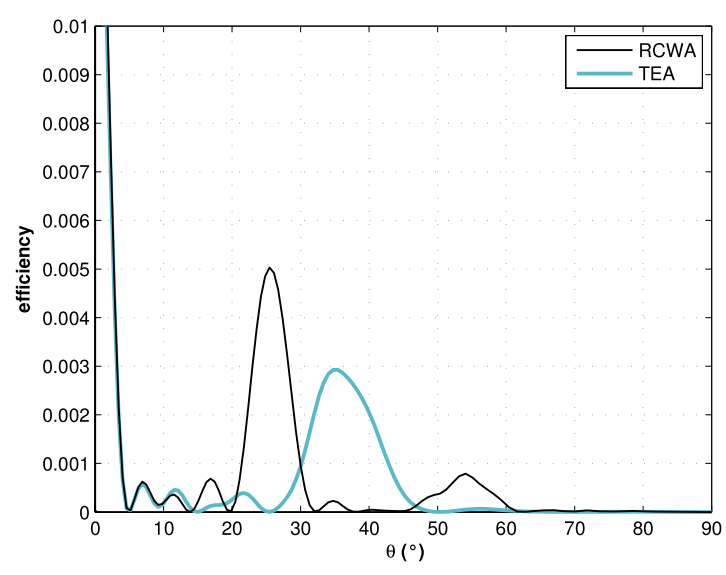

(a)

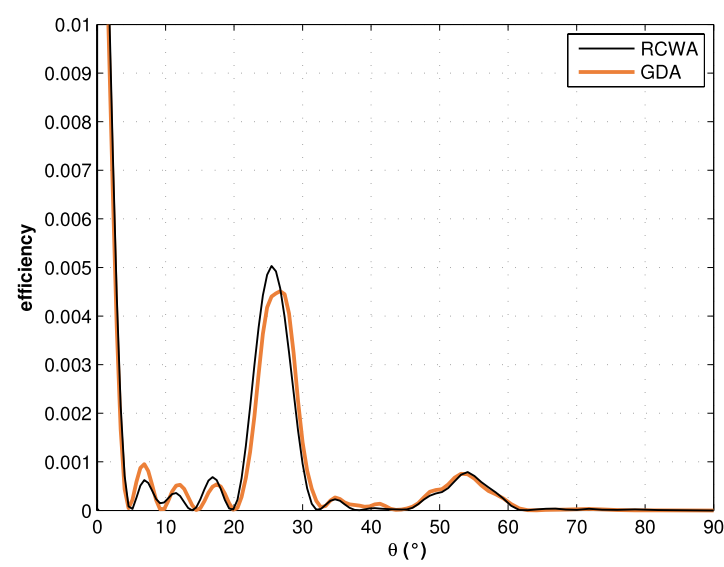

(b)

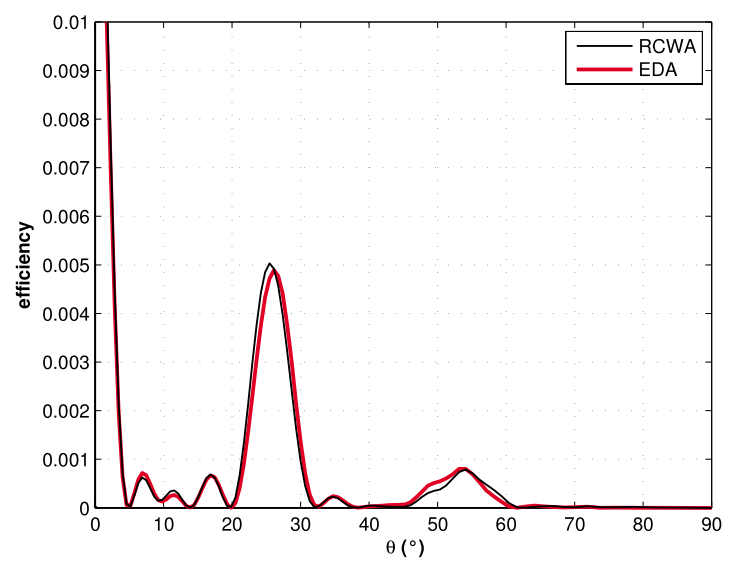

(c)

Fig. 5. (Color online) Diffraction efficiency as a function of diffraction angle for a structure with $h=6 \mu \mathrm{m}, L=6 \mu \mathrm{m}, n=1.3$, and $\Lambda=50 \mu \mathrm{m}$ computed with (a) TEA, (b) GDA, and (c) EDA models and compared with the RCWA model.

different from those of the RCWA method. For example, the diffraction maximum at $25^{\circ}$ does not appear in the TEA results. More generally, one can note that the larger differences between the two models occur at large diffraction angles. This corresponds to the fact that the differences between the output fields in the TEA and RCWA methods [Figs. 3(a) and 4(a)] have high spatial frequen- cies. Since the diffraction pattern is linked to the Fourier transform of this field, this corresponds to differences at larger diffraction angles.

In order to improve the performance of the scalar model, in the next section we shall take into account the physical depth of the structure.

\section{B. Geometrical Deep Element Approximation (GDA) Model}

In this section we take into account the physical depth of the structure by considering geometrical optics effects, i.e., ray tracing [2]. Hazra et al. [17] already accounted for thickness effects in a diffracting triangle based on their geometrical optics description. In order to compute the output field, we consider that light is reflected and refracted on the sides of the triangle (Fig. 6). This model will be called the geometrical deep element approximation (GDA) model. For the sake of simplicity, we shall assume that light is only reflected or refracted one time. This means that we do not consider the multiple reflections that may occur inside the triangle after refraction on the side. The consequences of this simplification will be considered in Subsection 3.B.

As can be seen in Fig. 6, the field at the output of the structure can be decomposed in different contributions: $E_{\text {out }}$ is due to the light transmitted in air without interaction with the structure, $E_{t l}^{\mathrm{GDA}}(x)\left(E_{t r}^{\mathrm{GDA}}(x)\right)$ is the field induced by the rays refracted on the left (right) side of the triangle, and $E_{r l}^{\mathrm{GDA}}(x)\left(E_{r r}^{\mathrm{GDA}}(x)\right)$ is the field induced by the rays reflected on the right side of the triangle. The output field is equal to the sum of all of these contributions.

For symmetry reasons, one has $E_{t l}^{\mathrm{GDA}}(x)=E_{t r}^{\mathrm{GDA}}(-x)$ and $E_{r l}^{\mathrm{GDA}}(x)=E_{r r}^{\mathrm{GDA}}(-x)$. The fields $E_{t r}^{\mathrm{GDA}}(x)$ and $E_{r r}^{\mathrm{GDA}}(x)$ can be written in the form $E_{t r}^{\mathrm{GDA}}(x)=t \exp \left[i \phi_{t r}(x)\right]$ and $E_{r r}^{\mathrm{GDA}}(x)$ $=r \exp \left[i \phi_{r r}(x)\right]$ where $\phi_{t r}(x)$ and $\phi_{r r}(x)$ are the phase delays undergone by the light through propagation in the structure. Values $t$ and $r$ are the Fresnel transmission and reflection coefficients corresponding to the interfaces traversed by light (see Fig. 7 for notation):

$$
\begin{gathered}
r=\frac{n_{\text {air }} \cos \alpha-n \cos \gamma}{n_{\text {air }} \cos \alpha+n \cos \gamma}, \\
t=\frac{2 n_{\text {air }} \cos \alpha}{n_{\text {air }} \cos \alpha+n \cos \gamma} .
\end{gathered}
$$

According to the notation in Fig. 7, and after elementary geometrical calculations, $\phi_{t r}(x)$ and $\phi_{r r}(x)$ can be expressed as

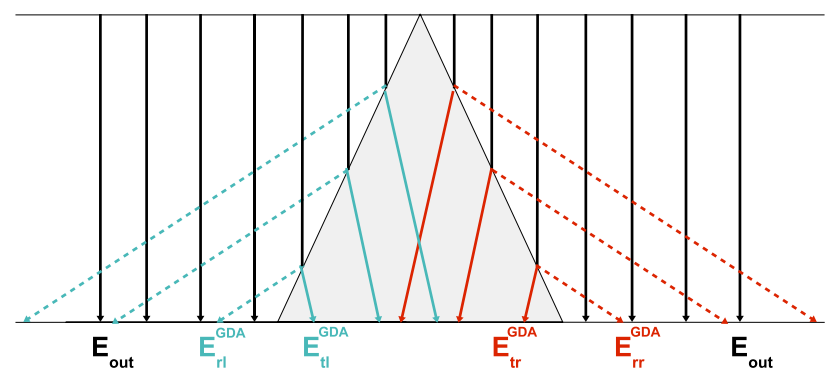

Fig. 6. (Color online) Different contributions to the output field in the GDA model. 

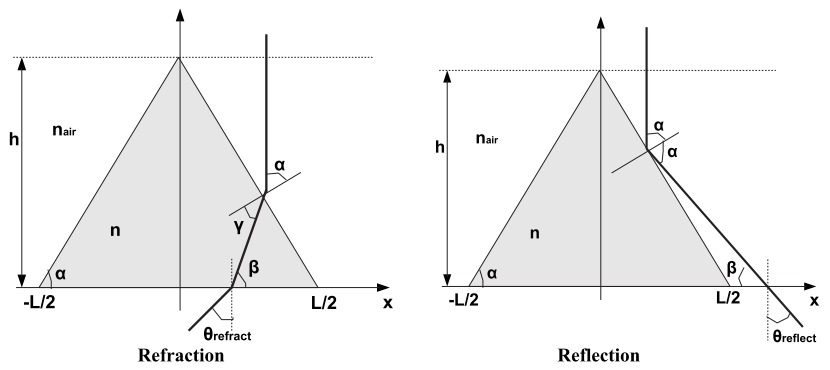

Fig. 7. Refraction and reflection of light on the right side of the structure.

$$
\begin{aligned}
\phi_{t r}(x)= & \frac{2 \pi}{\lambda}\left[\frac{\tan \alpha}{\tan \alpha+\tan \beta}\left(n_{\text {air }} \tan \beta-\frac{n}{\cos \beta}\right) x\right. \\
& +\frac{\tan \alpha}{\tan \alpha+\tan \beta} \frac{L}{2}\left(n_{\text {air }} \tan \alpha+\frac{n}{\cos \beta}\right) \\
& \left.-\frac{l}{2} n_{\text {air }} \tan \alpha\right], \\
\phi_{r r}(x)= & \frac{2 \pi}{\lambda}\left[n_{\text {air }} \frac{\tan \alpha}{\tan \beta-\tan \alpha}\left(\tan \beta-\frac{1}{\cos \beta}\right) x\right. \\
& +\frac{L}{2} \frac{\tan \alpha}{\tan \beta-\tan \alpha} n_{\text {air }}\left(-\tan \alpha+\frac{1}{\cos \beta}\right) \\
& \left.-\frac{l}{2} n_{\text {air }} \tan \alpha\right] .
\end{aligned}
$$

The amplitude and the phase of the output field calculated using this model are represented, respectively, in Figs. 3(b) and 4(b). One can notice a significant improvement with respect to the TEA model. In particular, the oscillations of the amplitude are well represented inside the structure and in the outside region closer to the structure where the rays transmitted in air and the rays reflected on the side of the triangle interfere.

The far-field diffraction pattern is displayed in Fig. 5(b). Results are much closer to RCWA results than for the TEA model. In particular, it can be noticed that the positions and the amplitudes of the two maxima of intensity $\left(\theta=25^{\circ}\right.$ and $\left.\theta=53^{\circ}\right)$ are correctly predicted by the GDA model. It can be shown that the direction of the first maximum corresponds to the angle of refraction of the incident rays through the structure (denoted by $\theta_{\text {refract }}$ in Fig. 7). The direction of the second maximum corresponds to the ray reflected by the side of the triangle (denoted by $\theta_{\text {reflect }}$ in Fig. 7). It is clear that these two directions cannot be predicted by the TEA model, which does not take into account the depth of the structure. In order to quantify the error induced by this model, in Table 1 we have given the root mean square deviation (RMSD) between the diffraction efficiencies of the different models and the RCWA result. RMSD is computed according to Eq. (8) where $\eta_{i}^{\mathrm{GDA}}$ and $\eta_{i}^{\mathrm{RCWA}}$ are the efficiencies of the ith diffraction order computed with the GDA and RCWA models and the $n$ diffraction order corresponding to a diffraction angle of $90^{\circ}$ respectively. It has to be noted that we have not taken into account the order 0 in the expression of
Table 1. RMSD of the Diffraction Efficiencies of the Different Models from the RCWA Solution Computed for Different Structures of Index $n=1.3$

\begin{tabular}{cccc}
\hline & TEA & GDA & EDA \\
\hline $\begin{array}{c}h=L=6 \mu \mathrm{m} \\
\Lambda=50 \mu \mathrm{m}\end{array}$ & $1.9 .10^{-3}$ & $3.86 .10^{-4}$ & $2.82 .10^{-4}$ \\
$\begin{array}{c}h=L=2 \mu \mathrm{m} \\
\Lambda=16.67 \mu \mathrm{m}\end{array}$ & $1.9 .10^{-3}$ & $1.7 .10^{-3}$ & $3.85 .10^{-4}$ \\
$h=L=0.5 \mu \mathrm{m}$ & $3.9 .10^{-3}$ & $3.7 .10^{-3}$ & $3.5 .10^{-3}$ \\
$\Lambda=4.17 \mu \mathrm{m}$ & & & \\
\hline
\end{tabular}

RMSD. It is seen in the first line of Table 1 that the RMSD of GDA is 5 times smaller than that of the TEA.

$$
\operatorname{RMSD}=\sqrt{\frac{1}{n} \sum_{i=1}^{n}\left(\eta_{i}^{\mathrm{RCWA}}-\eta_{i}^{\mathrm{GDA}}\right)^{2}}
$$

As can be seen in Fig. 3(b) and as is clear from its principle, the field given by the GDA model presents some discontinuities. These do not occur in the RCWA model. In order get closer to the RCWA model, in the next section we will take into account the diffraction due to the spatial limitation induced by the sides of the triangle.

\section{Edge Diffraction Approximation (EDA) Model}

In the GDA model, the field at the output of the structure is decomposed as the sum of different contributions due to reflection and refraction on the sides of the triangle. This approach is purely based on geometrical optics, and it does not take into account the fact that each contribution is spatially limited by the side of the triangle. In reality, however, the spatial limitation produces diffraction.

In this section, this diffraction phenomenon will be modeled by considering the diffraction by the edges of the triangle. These edges are geometrically equivalent to knife edges, a classical problem in diffraction theory. This model will therefore be called the edge diffraction approximation (EDA). A similar model was used by Ufimtsev [12] in his Fundamentals of the Physical Theory of Diffraction to approximate the scattering from perfectly conducting bodies. In the EDA model as in the GDA model, the output field is given by the addition of the different contributions. For symmetry reasons, we only detail the contributions of the right side of the triangle (Fig. 8 ). Since we are interested in the electrical field on the $x$

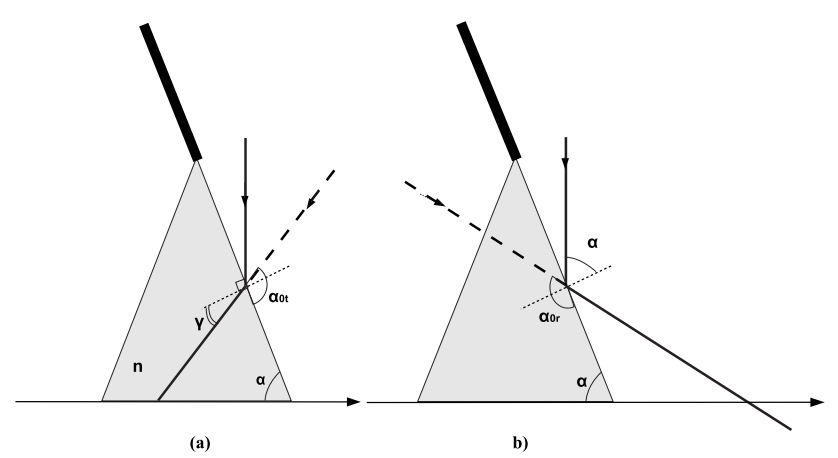

Fig. 8. EDA model: refracted and reflected field from the right side of the triangle. The thick black line represent the knife edge. 
axis at the base plane of the triangle, diffraction by the lower knife edge will be neglected and we shall only consider the half-plane diffraction by the upper edge, schematically depicted as a virtual knife edge in the figure. However, the side of the triangle contains a second feature: in addition to the knife edge, it is also a refracting diopter. To take into account the refraction at the index discontinuity between air and the structure of index $n$, we consider the diffraction phenomenon to occur in an homogeneous medium of index $n$ as far as the refracted wave contribution is concerned [Fig. 8(a)], while for the reflected wave contribution, diffraction in the air is considered [Fig. 8(b)].

Applying Kirchhoff's boundary conditions ([18], Section 8.3.2) at the knife edge, with the field considered to vanish on the knife edge and to remain unchanged in the rest of the knife edge's plane, leads to the classical Cornu's spiral method. We selected the solution developed in [18], Section 11.5; this method is rigorous for a semi-infinite perfectly conducting knife edge and provides a solution everywhere in space. We considered it as a working approximation for our case where we need the field at the base plane of the triangle, which lies obliquely to the knife edge and intersects it at point $x=L / 2$. Under these assumptions we used the result in [18], which states that the field at the point $M(r, \vartheta)$ diffracted by a perfectly conducting knife edge $X<0$ is given by

$$
E_{\alpha_{0}}^{n}(r, \vartheta)=\frac{e^{-i \pi / 4}}{\sqrt{2}} e^{2 i \pi n r / \lambda} e^{-i \pi \xi^{2} / 2}\left[\frac{1}{2}+C(\xi)+i\left(\frac{1}{2}+S(\xi)\right)\right]
$$

with

$$
\xi=\sqrt{\frac{8 n r}{\lambda}} \cos \left(\frac{\vartheta-\alpha_{0}}{2}\right),
$$

where $\alpha_{0}$ is the angle between the incident ray and the knife edge, $n$ the optical index of the medium, $(r, \vartheta)$ the polar coordinates (see Fig. 9), and $C(a)$ and $S(a)$ the Fresnel integrals defined by

$$
C(a)=\int_{0}^{a} \cos \left(\frac{\pi t^{2}}{2}\right) \mathrm{d} t
$$

and

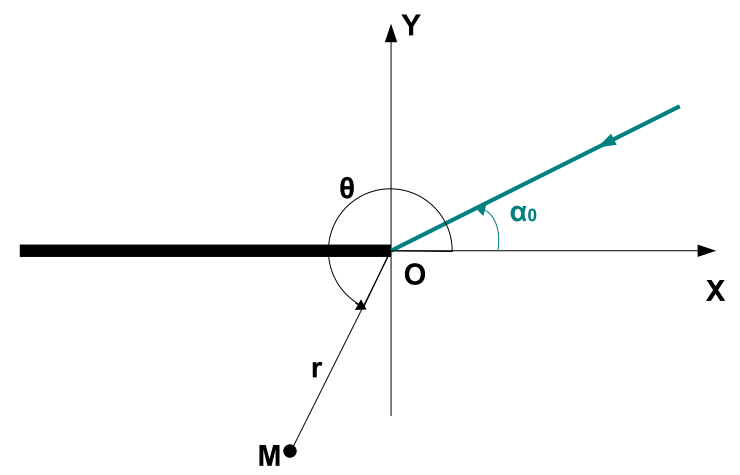

Fig. 9. (Color online) Reference axes for diffraction by a knife edge.

$$
S(a)=\int_{0}^{a} \sin \left(\frac{\pi t^{2}}{2}\right) \mathrm{d} t
$$

The refractive contribution $E_{t r}^{\mathrm{EDA}}(x)$ is thereby modeled as the field diffracted by a knife edge illuminated with a plane wave with incidence $\alpha_{0 t}=\pi / 2+\gamma$ in a medium of in$\operatorname{dex} n$ :

$$
E_{t r}^{\mathrm{EDA}}(x)=t E_{\alpha_{0 t}}^{n}\left(\sqrt{h^{2}+x^{2}}, \tan ^{-1}\left(\frac{x}{h}\right)-\tan ^{-1}\left(\frac{L}{2 h}\right)\right),
$$

where $t$ is the transmission Fresnel coefficient through the interface between media of index $n_{\text {air }}$ and $n$ with an incidence angle equal to $\alpha$ [see Fig. 8(a)].

In the same manner, the reflective contribution $E_{r r}^{\mathrm{EDA}}(x)$ is modeled as the field diffracted by a knife edge illuminated by a plane wave with incidence $\alpha_{0 r}=\pi / 2+\alpha$ in a medium of index $n_{\text {air }}$ [see Fig. 8(b)]:

$$
E_{r r}^{\mathrm{EDA}}(x)=r E_{\alpha_{0 r}}^{n_{\text {air }}}\left[\sqrt{h^{2}+x^{2}},-\tan ^{-1}\left(\frac{x}{h}\right)+\tan ^{-1}\left(\frac{L}{2 h}\right)\right],
$$

where $r$ is the reflection Fresnel coefficient on the interface between media of index $n_{\text {air }}$ and $n$ with an incidence angle equal to $\alpha$.

Besides the $E_{t r}^{\mathrm{EDA}}$ and $E_{r r}^{\mathrm{EDA}}$ contributions, we have to add to the EDA model the contributions of the left side of the triangle and the contribution $E_{\text {out }}$ of the field that do not interact with the triangular structure. Due to symmetry reasons, the contributions of the left side of the triangle can be deduced from the right side contributions: $E_{t l}^{\mathrm{EDA}}(x)=E_{t r}^{\mathrm{EDA}}(-x)$ and $E_{r l}^{\mathrm{EDA}}(x)=E_{r r}^{\mathrm{EDA}}(-x)$.

The field obtained with the EDA model is plotted in Figs. 3(c) and 4(c). It is seen to be closer to the RCWA result that the GDA model. With this model the problem of discontinuity of the field inside and outside the triangle is solved. In particular, there is no longer a discontinuity at $x=8 \mu \mathrm{m}$, and the oscillations at a long distance of the structure are correctly represented. This result shows that these oscillations stem from Fresnel diffraction at the edges of the triangular structure. However, discontinuities at $x=-L / 2$ and $x=L / 2$ that are barely visible in Fig. 3 still remain. The resulting diffraction pattern is plotted in Fig. 5(c). It is seen to fit better to the RCWA results than the GDA model, especially for angles between $5^{\circ}$ and $30^{\circ}$.

The results show that most details of the field and of the diffraction pattern are given by the GDA model that takes into account the depth of the element in a purely geometrical way. This conclusion can also be corroborated from Table 1 where the decrease of RMSD from GDA to EDA is modest. In conclusion, taking into account the diffractive, Fresnel-like effects as in the EDA model leads to slight improvement and reveals some details of the field that did not appear with the GDA model. 


\section{LIMITS OF THE MODEL}

In this section we analyze some limitations of our model. We first study its performance as the size of the structure decreases. We then consider the effect of internal reflections inside the triangular structure.

\section{A. Critical Size of the Structure}

Scalar approximation is known to agree with reality when the feature size of the structure is sufficiently large compared with the wavelength [3]. Until now, the structure considered in this paper had a feature size 8 times larger than the wavelength. In this section we consider smaller feature sizes and study how the results are modified. We shall consider two structures with decreasing feature sizes. We first study a triangular structure with $L$ $=2 \mu \mathrm{m}$ and $h=2 \mu \mathrm{m}$, and then a smaller structure with $L=0.5 \mu \mathrm{m}$ and $h=0.5 \mu \mathrm{m}$, which corresponds to a feature size equal to the wavelength. For these two structures, the index $n$ inside the triangle is equal to 1.3 (same value as in previous sections) and the periodicity is chosen so

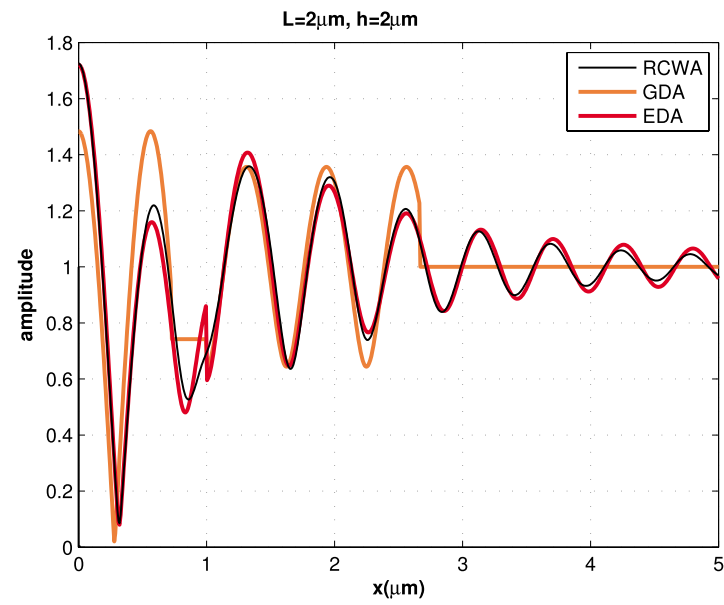

(a)

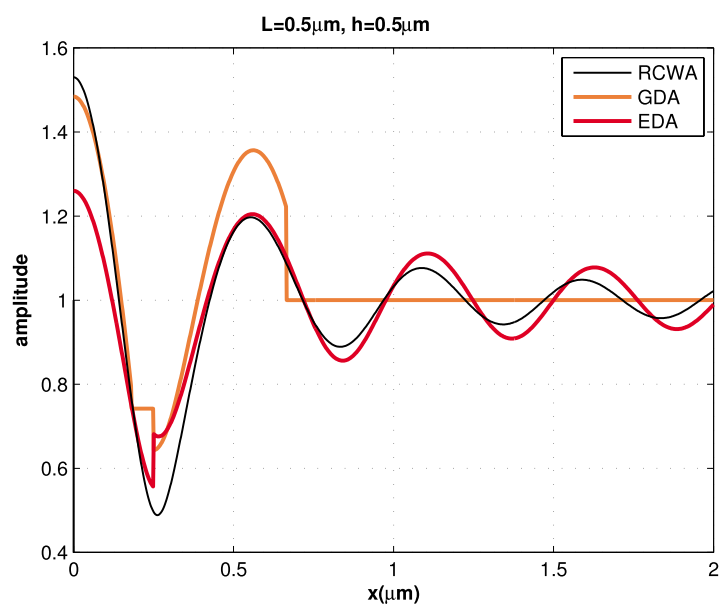

(b)

Fig. 10. (Color online) Amplitude of the field computed with the GDA, EDA, and RCWA models for two components: (a) $L=2 \mu \mathrm{m}$, $h=2 \mu \mathrm{m}$, and $\Lambda=16.67 \mu \mathrm{m}$; (b) $L=0.5 \mu \mathrm{m}, h=0.5 \mu \mathrm{m}$, and $\Lambda$ $=4.17 \mu \mathrm{m}$. that all of the compared components here have the same fill factor (i.e., the same ratio $L / \Lambda$ ). Thereby, as done previously we chose a periodicity of $\Lambda=50 \mu \mathrm{m}$ for the structure with $L=6 \mu \mathrm{m}$; the periodicities of the structures with $L=2 \mu \mathrm{m}$ and $L=0.5 \mu \mathrm{m}$ are equal to $16.67 \mu \mathrm{m}$ and $4.17 \mu \mathrm{m}$, respectively.

The amplitude and the phase of the field at the output of the structure computed with the GDA, EDA, and RCWA models and for each structure are represented in Figs. 10 and 11, and the diffraction pattern is represented in Fig. 12. In these figures we notice that the results given by the EDA model deviate from the results given by the RCWA increasingly as the feature size of the triangle decreases. Indeed, in Table 1, the RMSD of the diffraction efficiencies of the EDA model from the RCWA solution is equal to $2.84 .10^{-4}$ for the larger structure $(L=6 \mu \mathrm{m})$, to $3.85 .10^{-4}$ for the structure with $L=2 \mu \mathrm{m}$, and to $3.5 .10^{-3}$ for the structure with $L=0.5 \mu \mathrm{m}$. However, the global shape of curves given by the EDA model is still correct while the result given by the GDA model significantly departs from the RCWA results.

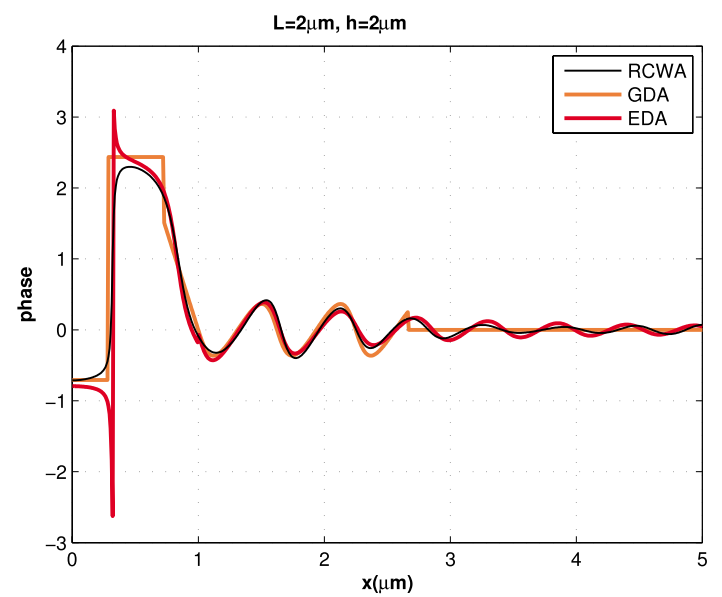

(a)

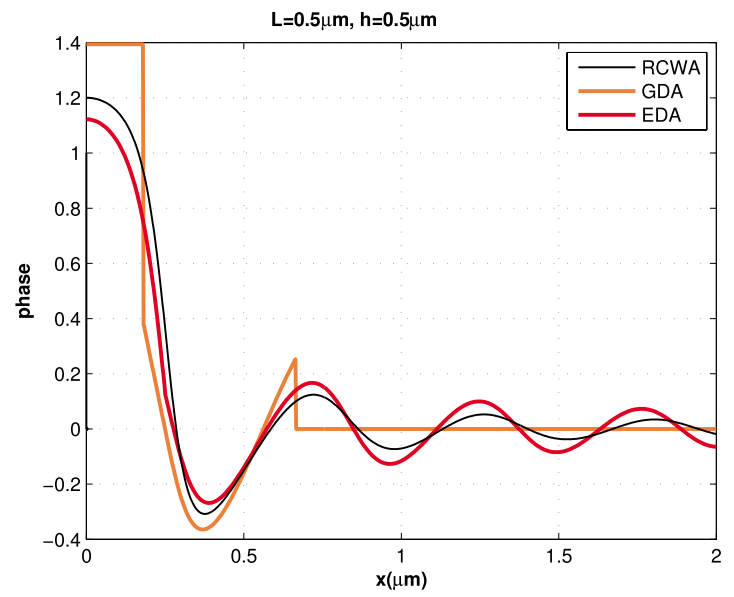

(b)

Fig. 11. (Color online) Phase of the field computed with the GDA, EDA, and RCWA models for two components: (a) $L=2 \mu \mathrm{m}$, $h=2 \mu \mathrm{m}$, and $\Lambda=16.67 \mu \mathrm{m}$; (b) $L=0.5 \mu \mathrm{m}, h=0.5 \mu \mathrm{m}$, and $\Lambda$ $=4.17 \mu \mathrm{m}$. 


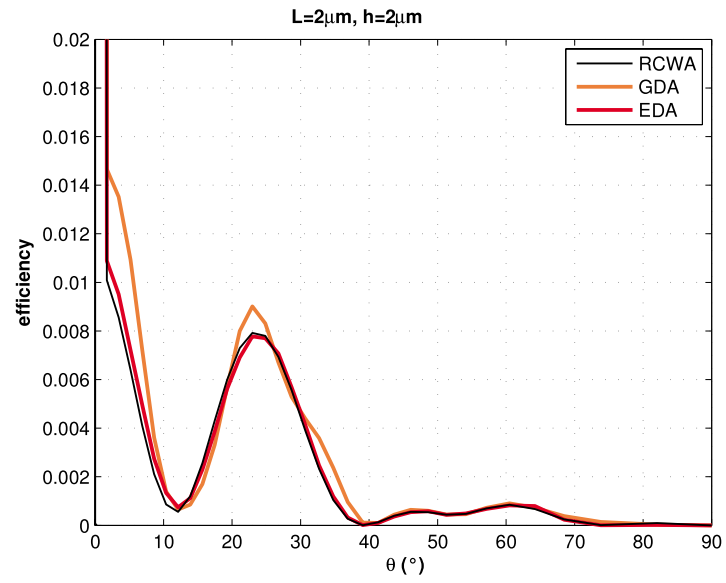

(a)

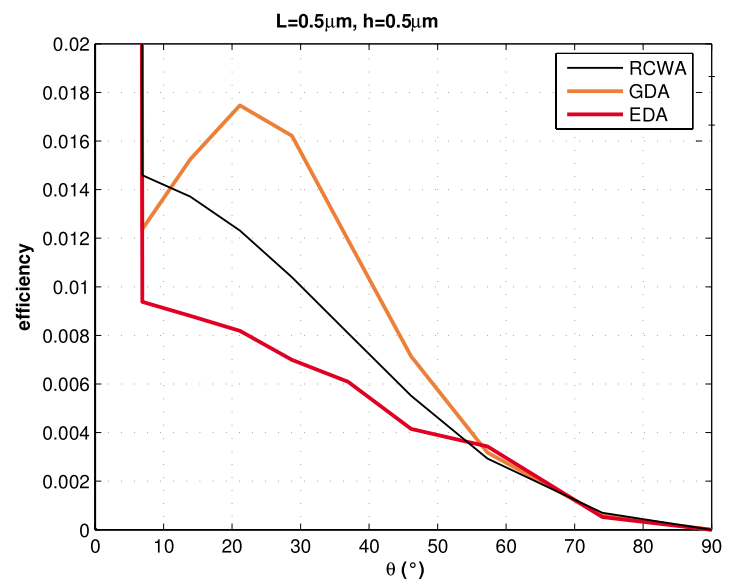

(b)

Fig. 12. (Color online) Diffraction efficiency as a function of diffraction angle computed with the GDA, EDA, and RCWA models for two components: (a) $L=2 \mu \mathrm{m}, h=2 \mu \mathrm{m}$, and $\Lambda=16.67 \mu \mathrm{m}$; (b) $L=0.5 \mu \mathrm{m}, h=0.5 \mu \mathrm{m}$, and $\Lambda=4.17 \mu \mathrm{m}$.

\section{B. Influence of the Structure Parameters and of} Reflections inside the Triangle

The scalar models developed in the previous section do not take into account the potential reflection of light inside the triangle (Fig. 13). In our models, the light re-

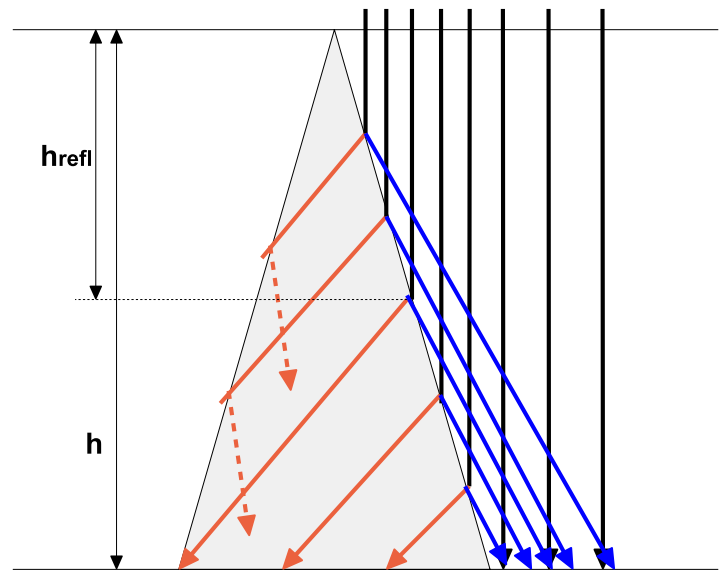

Fig. 13. (Color online) Reflection of light inside the triangular structure. flected inside the triangle is considered lost. Such multiple reflections appear when the structure has either a high refractive index or a steep slope. In order to quantify the light transmitted through the triangle that undergoes internal reflection, we define the ratio $f=h_{\text {refl }} / h$, where $h$ is the height of the triangle and $h_{\text {refl }}$ is linked to the quantity of geometrically transmitted rays that are reflected on the opposite side of the triangle (see Fig. 13). The ratio $f$ is equal to zero when no geometrical rays are reflected and is equal to unity when all the rays are reflected. In order to illustrate the influence of this effect, we have chosen a structure with $L=4 \mu \mathrm{m}, h=6.5 \mu \mathrm{m}$, and $n=1.4$. In this case, the ratio $f$ is equal to 0.3 .

As we have seen previously that the EDA model is the scalar model most representative of reality, we chose in this part to represent only the results given by the EDA model and the RCWA model. The amplitude and the phase of the field at the output of the structure are represented in Fig. 14 and the diffraction pattern is represented in Fig. 15. It can be noticed that under the triangle, that is, for $x \in[-L / 2, L / 2]$, the field given by the EDA model differs significantly from the field given by the RCWA model. On the diffraction pattern, this difference appears for small angles.

Taking into account this reflection inside the triangle that appears for certain structures would make our scalar

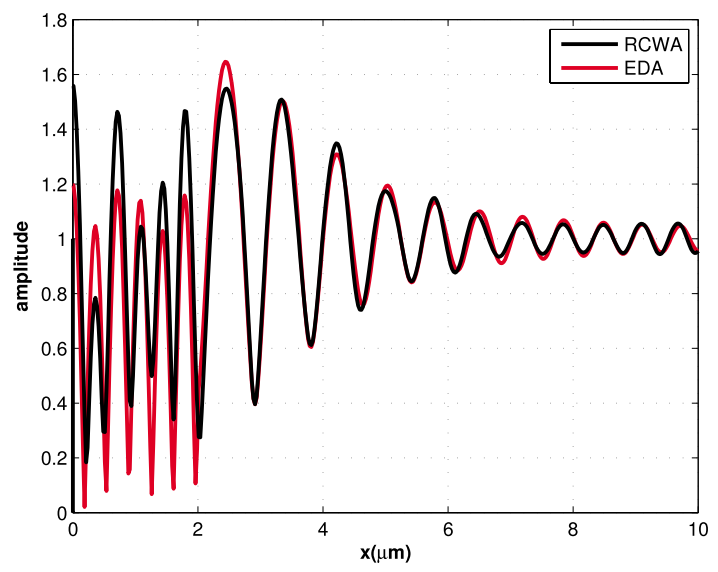

(a)

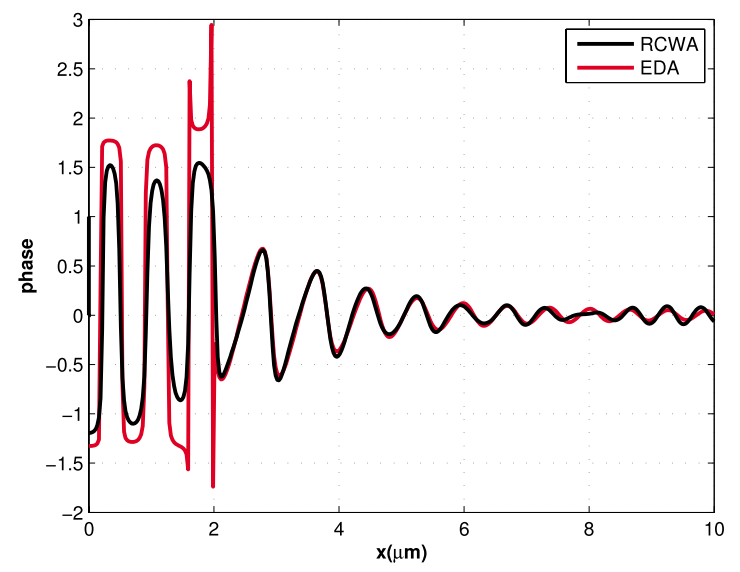

(b)

Fig. 14. (Color online) Amplitude and phase of the field for a structure with $h=6.5 \mu \mathrm{m}, L=4 \mu \mathrm{m}$, and $n=1.4$. 


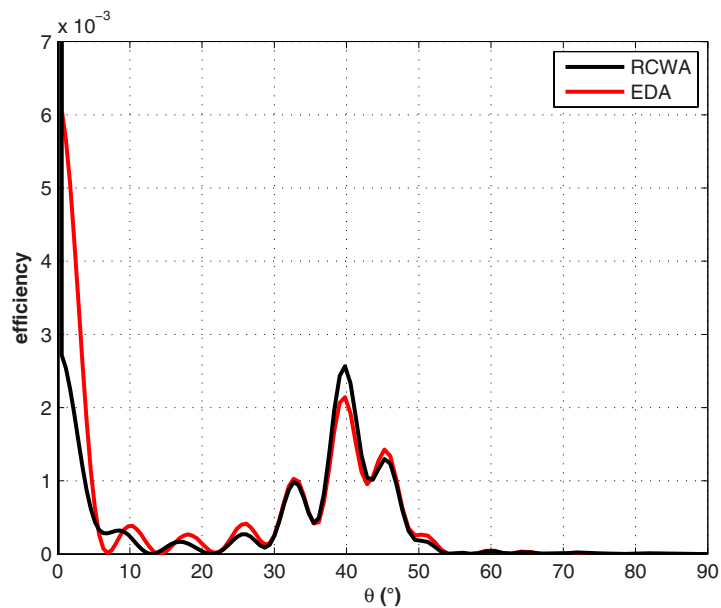

Fig. 15. (Color online) Diffraction pattern for a structure with $h=6.5 \mu \mathrm{m}, L=4 \mu \mathrm{m}$, and $n=1.4$.

model even more complex. Since it is our whole purpose with this article to build a simple scalar model that can describe the main features of the real diffraction pattern, we preferred not to complicate it any more, even though its scope of application is therefore limited.

\section{CONCLUSION}

In this article we have demonstrated that the diffraction pattern of a triangular structure illuminated with a plane wave with TE polarization can be well predicted by relatively simple scalar considerations. By scalar, we mean here that only one scalar quantity, in this case the electric field component perpendicular to the incidence plane, is sufficient for a good description of the diffraction phenomena in the near as well as in the far field. The main features of the diffraction pattern are obtained when taking into account the depth of the structure in a purely geometrical way. Some finer structures are grasped when taking into account the diffraction at discontinuities. This scalar model is still valid for small structures with feature sizes of the order of the wavelength but is limited to structures where no internal reflections occurs. Similar results can be obtained for TM polarization by only changing Fresnel transmission and reflection coefficients from TE to TM ones in GDA and EDA models.

This work has many perspectives. The generalization of the EDA model to oblique incidence is an interesting and challenging problem. Its application to other types of structure shapes is also interesting. It has recently been applied to blazed gratings in [19].

\section{ACKNOWLEDGMENTS}

During the initial phase of this work, D. Kuang was a Visiting Fellow at Laboratoire Charles Fabry de L'Institut
d'Optique. The authors thank J.-P. Hugonin, P. Lalanne, and J. Taboury for fruitful discussions.

\section{REFERENCES}

1. J. Turunen, "Diffraction theory of microrelief gratings," In Micro-optics. Elements, Systems and Applications, H. P. Herzig, ed. (Taylor \& Francis, 1997).

2. G. J. Swanson, "Binary optics technology: theoretical limits on the diffraction efficiency of multilevel diffractive optical elements," MIT Tech. Rep. 914 (1991).

3. D. A. Pommet, M. G. Moharam, and E. B. Grann, "Limits of scalar diffraction theory for diffractive phase elements," J. Opt. Soc. Am. A 11, 1827-1834 (1994).

4. J. M. Bendickson, E. N. Glytsis, and T. K. Gaylord, "Scalar integral diffraction methods: unification, accuracy, and comparison with a rigorous boundary element method with application to diffractive cylindrical lenses," J. Opt. Soc. Am. A 15, 1822-1837 (1998).

5. M. Testorf, "Perturbation theory as a unified approach to describe diffractive optical elements," J. Opt. Soc. Am. A 16, 1115-1123 (1999).

6. W. Singer and H. Tiziani, "Born approximation for the nonparaxial scalar treatment of thick phase gratings," Appl. Opt. 37, 1249-1255 (1998).

7. U. Levy, E. Marom, and D. Mendlovic, "Thin element approximation for the analysis of blazed gratings: simplified model and validity limits," Opt. Commun. 229 11-21 (2004).

8. O. Sandfuchs, R. Brunner, D. Pätz, S. Sinzinger, and J. Ruoff, "Rigorous analysis of shadowing effects in blazed transmission gratings," Opt. Lett. 31, 3638-3640 (2006).

9. W. Singer and K.-H. Brenner, "Transition of the scalar field at a refracting surface in the generalized Kirchhoff diffraction theory," J. Opt. Soc. Am. A 12, 1913-1919 (1995).

10. V. Kettunen, M. Kuittinen, and J. Turunen, "Effects of abrupt surface-profile transitions in nonparaxial diffractive optics," J. Opt. Soc. Am. A 18, 1257-1260 (2001).

11. N. Kerwien, T. Schuster, S. Rafler, W. Osten, and M. Totzeck, "Vectorial thin-element approximation: a semirigorous determination of Kirchhoff's boundary conditions," J. Opt. Soc. Am. A 24, 1074-1084 (2007).

12. P. Ya. Ufimtsev, Fundamentals of the Physical Theory of Diffraction (Wiley, 2007).

13. M. G. Moharam, E. B. Grann, D. A. Pommet, and T. K. Gaylord, "Formulation for stable and efficient implementation of the rigorous coupled-wave analysis of binary gratings," J. Opt. Soc. Am. A 12, 1068-1076 (1995).

14. P. Lalanne and G. M. Morris, "Highly improved convergence of the coupled-wave method for tm polarization," J. Opt. Soc. Am. A 13, 779-784 (1996).

15. J. W. Goodman, Introduction to Fourier Optics, 2nd ed., McGraw-Hill Physical and Quantum Electronics Series (McGraw-Hill, 1996).

16. E. N. Glytsis, "Two-dimensionally periodic diffractive optical elements: limitations of scalar analysis," J. Opt. Soc. Am. A 19, 702-715 (2002).

17. L. Hazra, Y. Han, and C. A. Delisle, "Geometric optical limits in the synthesis of planar kinoform lenses for axial stigmatism," Opt. Eng. 34, 1296-1302 (1995).

18. M. Born and E. Wolf, Principles of Optics (Pergamon, 1980).

19. H. Wang, D. Kuang, and Z. Fang, "Diffraction analysis of blazed transmission gratings with a modified extended scalar theory," J. Opt. Soc. Am. A 25, 1253-1259 (2008). 